\title{
Hard tissue formation induced by synthetic oligopeptide derived from an enamel matrix derivative
}

\author{
Michiko Noguchi ${ }^{1}$, Kazuya Tominaga ${ }^{2}$, Akio Tanaka $^{2}$, Masatoshi Ueda ${ }^{3}$ \\ ${ }^{\prime}$ Graduate School of Dentistry (Periodontology), Osaka Dental University, Hirakata, Japan. \\ ${ }^{2}$ Department of Oral Pathology, Osaka Dental University, Hirakata, Japan. \\ ${ }^{3}$ Department of Periodontology, Osaka Dental University, Hirakata, Japan.
}

Abstract

Background: Eosinophilic round bodies (ERBs) are composed of fragments of amino acid sequences of varying lengths, which represent a portion of exon 5 of amelogenin gene. ERBs have previously been shown to accumulate in the backs of rats subcutaneously injected with the product Emdogain ${ }^{\circ}$. In the present study, an oligopeptide with an amino acid sequence similar to this portion was artificially synthesized, and its effects were examined. Methods: The peptide solution was applied to periodontal defects created on the maxillae of rats. Sections from these tissues were histopathologically and immunohistochemically examined at 7 and 14 days after the start of the experiment. Results: In the experimental group, hard tissue had formed on the surface of exposed dentin, and both osteopontin and collagen type I (COLI) were localized around the hard tissue. The hard tissue was stained with periodic acid-Schiff (PAS) more deeply than was the dentin, and the fibers that were positive for COLI were vertically inserted in the rim of the newly-formed hard tissue. No hard tissue formation was found in the control group. Conclusion: These results suggest that synthetic oligopeptide administration can induce the formation of hard tissue, such as cementum, on the periodontal defects of rats.

[Oral Med Pathol 2012; 16: 75-80 doi: 10.3353/omp.16.75]

Key words: enamel matrix derivative, hard tissue formation, periodontal defects, rat, synthetic oligopeptides

Correspondence: Akio Tanaka, Department of Oral Pathology, Osaka Dental University, 8-1, Kuzuhahanazono-cho, Hirakata 573-1121, Japan

Phone: +81-72-864-3057, Fax: +81-72-864-3157, E-mail: tanaka@cc.osaka-dent.ac.jp

\section{Introduction}

Enamel matrix derivative (EMD, Emdogain ${ }^{\circledR}$, Biora AB, Malmö, Sweden), which has been widely used in clinical settings to repair damaged periodontal tissue (1), could possibly cause unexpected problems for humans, because of its animal (6-month-old pig)-derived preparations, though actual problematic phenomena have not yet reported (2). EMD contains various proteins, and it is not known which components of EMD modulate its biological effects (3-4). We showed that the old type of product EMD, which is packaged in two vials: (a vehicle solution and the freeze dried amelogenin fraction), induces the formation of eosinophilic round bodies (ERBs) and cartilage formation in the back of rats (4). However, newer products are provided as pre-mixed materials and are supposedly safer, because of their heat-treatment. Eosinophilic amorphous blobs, such as ERBs, are observed in rat periodontal defects after EMD application (5). ERBs derived from EMD may be formed via the self-assembly of amelogenin (6) and are composed of fragments of amino acid sequences of varying lengths (4). Each fragment (MW 1,118 Da) consists of a portion of exon 5 of amelogenin gene, as assessed using matrix-assisted laser desorption ionization time-of-flight mass spectrometry (MALDI-TOF MS) (4). An artificial oligopeptide with an amino acid sequence similar to this region was shown to induce the expression of genes related to osteogenesis in human periodontal ligament fibroblasts (7). In human gingival epithelial cells, the synthetic oligopeptide inhibited cell proliferation and adhesion, and promoted cell chemotaxis (8). Bone and cartilage formation and endochondral ossification were observed in the backs of rats injected with the synthetic oligopeptide together with an alginate impression material as a scaffold (6). In this study, the effects of the synthetic oligopeptide on artificial periodontal defects in rats were examined. 


\section{Materials and methods}

\section{Animals}

Twenty 7-week-old male Sprague-Dawley rats with an average weight of $230 \mathrm{~g}$ (Shimizu Laboratory Supplies Co., Ltd., Kyoto, Japan) were used in this study. Five rats each were used for the control and experimental groups. On 7 and 14 days after surgery in both groups, these groups of 5 rats were sacrificed as described below.

\section{Experimental protocol}

Rats were injected intraperitoneally with $0.3 \mathrm{mg} / \mathrm{kg}$ (body weight) of pentobarbital (Nembutal ${ }^{\circledR}$, Dainippon Sumitomo Pharma Co., Ltd., Osaka, Japan) after the administration of inhalation anesthesia with isoflurane (Abbott Japan Co., Ltd., Tokyo, Japan). An incision was made on the gingival sulcus between the palatal-distal site of the upper second molar and the middle of the mesial aspect of the upper first molar. Transverse 1-mm incisions were performed mesially from the middle of the mesial aspect of the upper first molar. After full-thickness flaps were raised, the alveolar bone, periodontal ligaments, and tooth roots were excised under irrigation using a round bur (ISO \#010, Maillefer Co., Tokyo, Japan) from the palatal-distal site of the upper second molar to the palatal-mesial aspect of the upper first molar. The deletions were carried out using a double-end-chisel (Ochsenbein \#3, Hu-Friedy, Chicago, IL, USA) and sufficient irrigation with normal saline. The synthetic oligopeptide (7) was prepared at a concentration of $15 \mathrm{mg} / \mathrm{ml}$ in $1.5 \%$ (w/v) propylene glycol alginate (PGA, Wako Pure Chemical Industries Ltd., Osaka, Japan) solution. The synthetic oligopeptide in PGA solution was then applied to the periodontal defects. As a control, the $1.5 \%$ PGA solution alone (without the synthetic oligopeptide) was applied to the periodontal defects. The full-thickness flaps were reinstated and sutured at the mesial side of the upper first molar using an absorbable suture thread (Biosorb C, Alcon Japan Ltd., Tokyo, Japan). The operative sites were astricted for 3 min using a swab. Rats were euthanized at 7 and 14 days after surgery, and were perfusion-fixed with $10 \%$ neutral buffered formalin (Nacalai Tesque Inc., Kyoto,
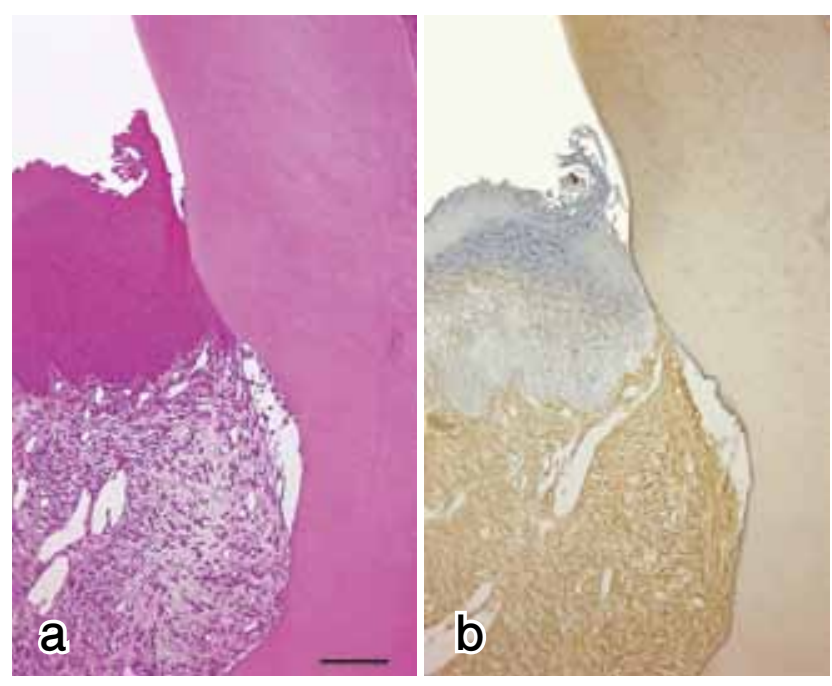

Japan). Periodontal tissues and the affected teeth were removed and immersed in fresh fixation buffer solution at $4^{\circ} \mathrm{C}$ for 3 days. The fixed tissues were decalcified with $10 \%$ ethylenediamine-tetraacetic acid solution (EDTA, Wako) at $4^{\circ} \mathrm{C}$ for approximately 1 month. The protocol for animal experiments was approved by the Animal Research Committee of Osaka Dental University (08-07003, 0902053, 10-03019).

\section{Sectioning and staining}

Formalin-fixed, paraffin-embedded sections were cut serially at $4 \mu \mathrm{m}$ and were stained with hematoxylin and eosin (HE). Sections were stained immunohistochemically with anti-osteopontin (LSL Co., Ltd., Tokyo, Japan) and anti-type I collagen (COLI) rabbit sera (LSL). Antigen retrieval was carried out by treatment with $2.5 \%$ hyaluronidase for $60 \mathrm{~min}$ at $37^{\circ} \mathrm{C}$ and $0.4 \%$ pepsin in $0.01 \mathrm{~N} \mathrm{HCl}$ for $30 \mathrm{~min}$ at $37^{\circ} \mathrm{C}$ for osteopontin and COLI, respectively. After the sections were washed, they were immersed in $3 \%$ hydrogen peroxide to block endogenous peroxidase activity (Nacalai Tesque Inc., Kyoto, Japan). Each antigen-antibody complex was detected using the EnVision+ system (Dako, Glostrup, Denmark) (9). Sections were also stained with toluidine blue (TB, concentration: $0.05 \%(\mathrm{w} / \mathrm{v}), \mathrm{pH} 7.0)$, periodic acid-Schiff (PAS) and Masson's trichrome (MT). Each section was examined using an optical microscope equipped with a digital camera (BX50, Olympus Optical Co., Ltd., Tokyo, Japan).

\section{Results}

\section{Control group}

The stratified squamous epithelium adhered to the tooth surface, whereas fiblobasts were present at a slightly higher density over the previously exposed root dentin 7 days after surgery. However, cementum-like hard tissue was not formed (Fig. 1a). COLI was expressed in the connective tissue and periodontal ligament (Fig. 1b). Stratified squamous epithelium, involving connective tissue and no formation of the cementum, was observed at day 14 after surgery (Fig. 2a). The previously formed artificial tissue

Fig. 1. Artificial periodontal defect from the control group at 7 days after surgery. (a) Hematoxylin and eosin (HE) stain; (b) immunoperoxidase stain for collagen type I (COLI), hematoxylin counterstain. $\mathrm{Bar}=100 \mu \mathrm{m}$. Many fibroblasts were present over the previously exposed root dentin (a). Immunoreactivity of COLI was observed in the gingival connective tissue (b). 
defects were repaired; they were composed of cellular and fibrous components at day 14 after surgery, in contrast with the results at day 7 after surgery (Fig. 2a).

Osteopontin was hardly expressed on the surface of the dentin (Fig. 2b). On the other hand, COLI was expressed in the connective tissue, and the staining intensity 14 days after surgery was similar to that observed 7 days after surgery (Fig. 2c).

For the TB staining, the dentin was stained cobalt (Fig. $2 \mathrm{~d}$ ), whereas the cementum and bone were stained less deeply than the dentin. However, none of the hard tissues from our specimens exhibited metachromatism for TB. The PAS staining was more intense in the cementum and bone tissue than in the dentin, although the cementum was not formed (Fig. 2e). The artificial defects adjacent to the dentin surface were filled with tissue consisting of collagen fibers, as visualized by blue MT staining (Fig. 2f).

\section{Experimental group}

Connective tissue at the surface of the defective dentin was observed 7 days after surgery (Fig. 3a). The cellularity tended to be higher than that of the control group. Newly formed hard tissue was observed on the surface of the defective dentin in rats and the interface between the newly formed hard tissue and the defective dentin was stained with hematoxylin 14 days after surgery (Fig. 3b).

The collagenous deposition at the surface of defective dentin did not contain osteopontin at 7 days after surgery (Fig. 3c), whereas at 14 days after surgery, osteopontin was expressed in cells involved in the newly formed hard tissue (Fig. 3d) and at the interface between the newly formed hard tissue and the dentin. The connective tissues were positive for COL1 at both 7 (Fig. 3e) and 14 days after surgery. The expression of COL1 was observed at the surface of the newly formed hard tissue (Fig. 3f). Collagen fibers that were positive for COL1 were vertically inserted into the newly formed hard tissue (Fig. 3f).

At both 7 and 14 days after surgery, none of the specimens exhibited metachromatism to TB (Figs. $3 g$ and 3h). The newly formed hard tissue was stained with TB less deeply (it appeared aqua in color) than the dentin at 14 days after surgery (Fig. 3h). The surface of the previously exposed dentin was not stained for PAS (Fig. 3i), although the junction between the newly formed hard tissue and the dentin was stained deeply with PAS (Figs. 3j). The newly formed hard tissue was also stained for PAS more deeply than was the dentin (Fig. 3j). The collagen deposition at the surface of the defective dentin was stained blue with MT at

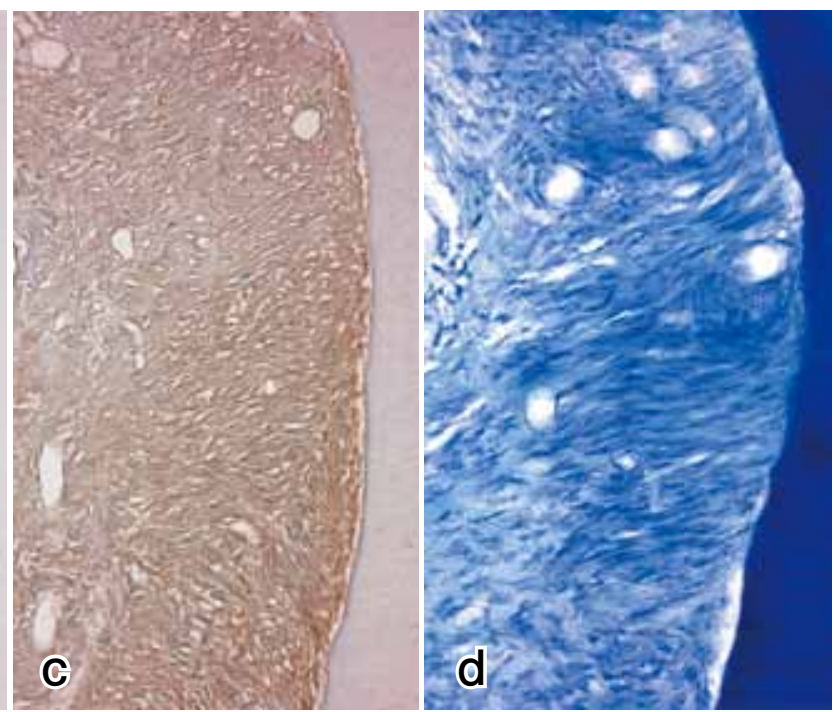

Fig. 2. Artificial periodontal defect from the control group at 14 days after surgery. (a) HE stain; immunoperoxidase stains for osteopontin (b) and COLI (c), hematoxylin counterstain; (d) toluidine blue (TB) stain; (e) periodic acid-Schiff (PAS) stain; (f) Masson's trichrome (MT) stain. Bar $=50 \mu \mathrm{m}$. Connective tissues were adhered to the dentin surface of an exposed root (a). Immunohistochemically, osteopontin was hardly expressed over the previously exposed dentin (b), while COLI could be observed in the periodontal ligament as well as in the gingival connective tissue (c). The dentin was stained a more intense cobalt than were the cementum and bone (d). The cementum was not formed on the surface of the exposed dentin (e). The artificial defect at the root dentin surface was filled with dense collagen fibers (f). 

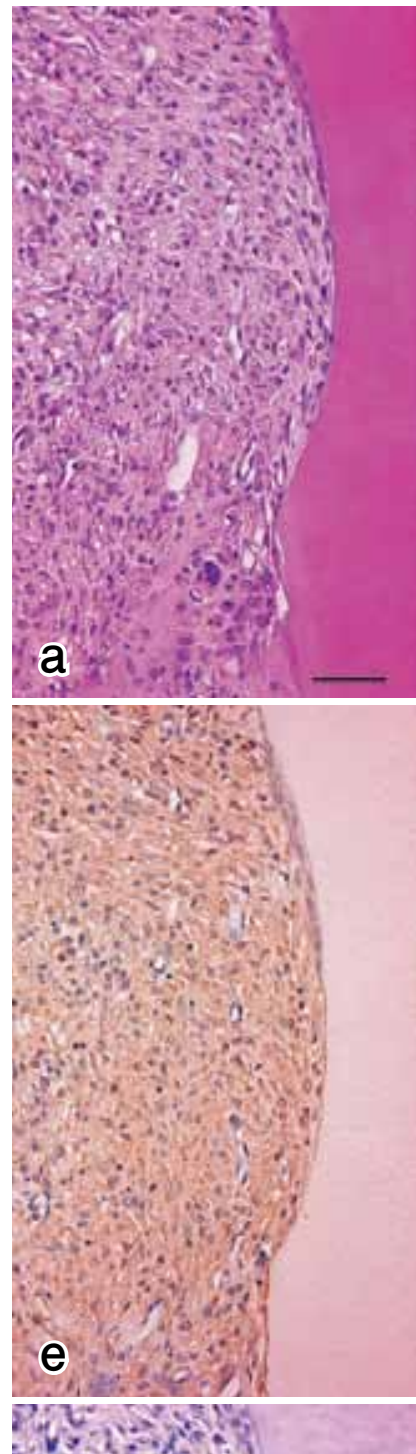

wex:-6it

Pा $20 \%$

and zals

on met

$35=8$.

Sinas an

zx es os

and

$8+2012$

2. $3,2: 4$

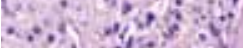

$2 \times 1 \%$

7.0 .

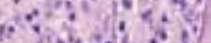

and nis.

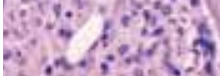

i $20 x$

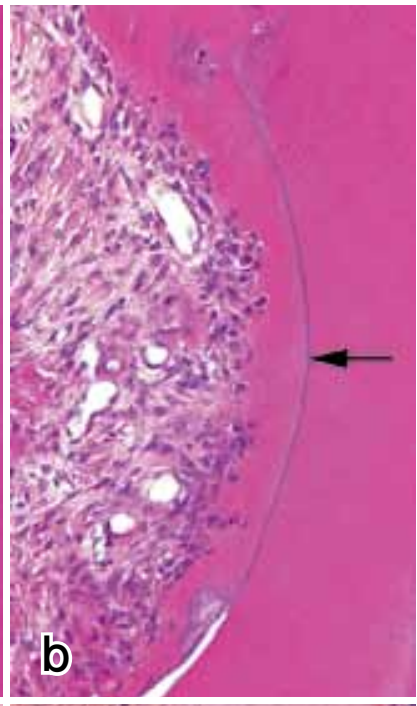

C
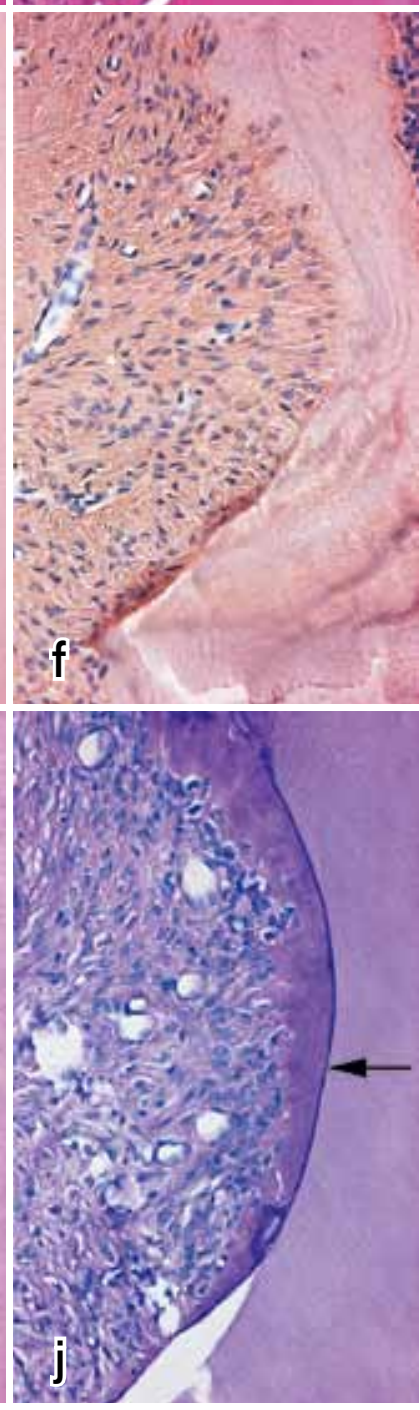
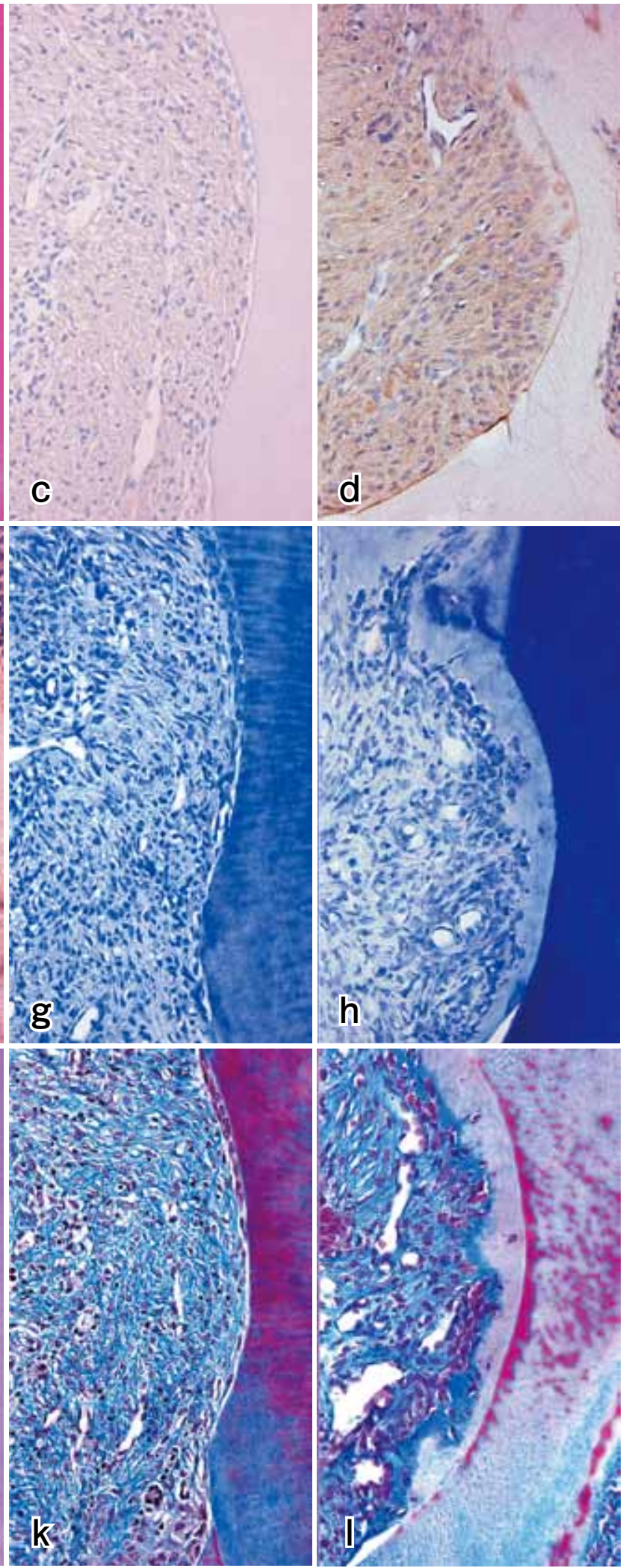

Fig. 3. Artificial periodontal defect of the experimental group at 7 days (a, c, e, g, i, k) and 14 days (b, d, f, h, j, l) after surgery. (a, b) HE stain; immunoperoxidase stains for osteopontin $(\mathbf{c}, \mathbf{d})$ and COLI $(\mathbf{e}, \mathbf{f})$, hematoxylin counterstain; $(\mathbf{g}, \mathbf{h})$ TB stain; (i, j) PAS stain; $(\mathbf{k}, \mathbf{l})$ MT stain. Bar $=50 \mu \mathrm{m}$. No newly formed hard tissues were present over the previously exposed root dentin surface on day 7 after surgery (a), but on day 14 hard tissues were observed (b). The interface (arrow) between the newly formed hard tissue and the defective dentin was stained with hematoxylin (b). Immunoreactivity of osteopontin was not observed in the repaired connective tissue on day 7 after surgery (c), but this was observed in the newly formed hard tissue on day 14 after surgery (d). The immunoreactivity of COLI was seen in the connective tissue on days 7 (e) and 14 after surgery. COLI-positive fibers were vertically inserted into the newly formed hard tissue (f). None of specimens exhibited metachromasia on day 7 after surgery $(\mathrm{g})$, and on day 14 after surgery newly formed hard tissue over the dentin was lightly stained (h). The surface of the defective dentin was lightly stained on day 7 after surgery (i), although the junction (arrow) between the newly formed hard tissue and dentin was deeply stained on day 14 after surgery (j). The collagenous deposition on the surface of defective dentin was stained blue $(\mathrm{k})$, and on day 14 after surgery the fibers that were stained blue vertically were inserted into the newly formed hard tissue (1). 
7 days after surgery (Fig. 3k). The fibers stained blue by MT were vertically oriented in the newly formed hard tissue at 14 days after surgery (Fig. 31).

\section{Discussion}

Complete regeneration of the gingival crevicular epithelium and the attached epithelium requires 14 days after surgical intervention to restore a normal structure to rat periodontal defects (10). Our study also found that marginal gingivae and gingival sulci were regenerated at 14 days after surgery.

Osteopontin, which is a phosphorylated glycoprotein, is produced at early stages of bone formation (11) and is expressed in cementoblasts lining the surface of tooth roots rather than in periodontal ligaments (12). The literature describes the immunohistochemical staining of the D-CJ with osteopontin $(13,14)$; in the current study, osteopontin staining weakly localized to the D-CJ and cementocytes. The surface tissue of the defective dentin expressed COLI at 7 days after surgery, although the expression of both osteopontin and COLI was observed at the surface of the newly formed hard tissue at 14 days after surgery. Moreover, at 14 days after surgery, osteopontin was expressed in cells involved in the newly formed hard tissue and at the site between this hard tissue and the dentin. These findings are compatible with the results of previous studies, which suggests that cementoblasts express both osteopontin (11, 15-16) and COLI $(11,16-18)$ during cell differentiation and matrix production. At 14 days after surgery, fibers that were positive for COLI, including those in the periodontal ligament (15), were vertically oriented in the newly formed hard tissue, which is indicative of the formation of a reattachment between the newly formed hard tissue and the connective tissue.

The application of EMD to the periodontal defects in rats led to the formation of acellular and cellular cementum more than 2 weeks after surgery (19). The artificial synthetic oligopeptide, which formed new hard tissue on the rat periodontal defects, was examined in this study. The newly formed hard tissue was stained an aqua color by TB, and was positive for PAS at 14 days post surgery. The staining pattern of the hard tissue resembled that of cementum rather than that of the dentin, and it seems as if dentin contained a lower proportion of polysaccharides compared with cementum and bone tissues $(13,20)$, because the cementum and bone tissue were stained by PAS more deeply than was the dentin.

Newly formed cementum shows metachromasia to TB (13, 20-21), whereas the hard tissues formed in our experiment did not show metachromasia to TB. This is thought to be due to the $\mathrm{pH}$ of the reaction and the decalcification method used. As the presence of cementum in the extracellular matrix is a characteristic of chondroitin sulfate glycosaminoglycan (22), cementum might be thought to show metachromasia by TB. A TB solution at $\mathrm{pH} 5.0$ was used for the staining in a previous study (21), which suggests that the discrepancy observed here was likely caused by the $\mathrm{pH}$ of the TB solution that was used ( $\mathrm{pH}$ 7.0). The use of an
EDTA solution or a formic-citrate acid solution is suitable for decalcification over a long period of time (23). A formiccitrate acid solution was used for long-term decalcification in the previous experiments that showed metachromasia to TB (20-21), whereas a neutral pH EDTA buffer solution was used for decalcification in our study. As the $\mathrm{pH}$ values of both the TB solution and the decalcification solution were neutral, metachromasia to TB may not have been evident in this study.

In a previous surgical protocol $(5,24)$, longitudinal incisions were performed at the maxillae of rats following the transverse incision, although a longitudinal incision was included in this study, in an attempt to reduce bleeding. A $1.5 \%$ PGA solution was used as a scaffold in this study. In an exploratory experiment, PGA remained insoluble in water, although a $6 \% \mathrm{~W} / \mathrm{W}$ (or $6 \% \mathrm{~W} / \mathrm{V}$ ) PGA solution was designed for use in studies performed in vivo (2). As the $0.6 \%(1 \% \mathrm{~W} / \mathrm{V})$ PGA (Wako) solution had a low viscosity, we decided to use a $1.5 \% \mathrm{~W} / \mathrm{V}$ PGA solution as the scaffold in this study (data not shown).

In summary, it is suggested that the administration of synthetic oligopeptide aids in the formation of hard tissues, such as cementum, in the periodontal defects of rats.

\section{Acknowledgments}

This study was supported in part by a Grant-in-Aid for Scientific Research (c) (No. 20592442) and by the HighTech Research Center Project for Private Universities: Matching Fund Subsidy from MEXT (the Japanese Ministry of Education, Cultures, Sports, Science and Technology), 2007-2011.

\section{References}

1. Silvestri M, Rasperini G, Milani S. 120 infrabony defects treated with regenerative therapy: long term results. $J$ Periodontol 2011; 82: 668-75.

2. Hammarström L, Heijl L, Gestrelius S. Periodontal regeneration in a buccal dehiscence model in monkeys after application of enamel matrix proteins. J Clin Periodontol 1997; 24: 669-77.

3. Gestrelius S, Andersson C, Lidström D, Hammarström L, Somerman M. In vitro studies on periodontal ligament cells and enamel matrix derivative. J Clin Periodontol 1997; 24: 685-92.

4. Kim N, Tominaga K, Tanaka A. Analysis of eosinophilic round bodies formed after injection of enamel matrix derivative into the backs of rats. $J$ Periodontol 2005; 76: 1934-41.

5. Fukunaga T, Noguchi Y, Imai H. Influence of enamel matrix derivative on early healing events in rat periodontal defects. Shika Igaku (J Osaka Odontol Soc) 2003; 66: 9-24. (Japanese)

6. Hida $\mathrm{T}$, Tominaga $\mathrm{K}$, Tanaka A. Tissue reaction to synthetic oligopeptide derived from enamel matrix derivative in rats. Oral Sci Inter 2010; 7: 26-33.

7. Kawanaka A, Tominaga K, Tanaka A. Effect of peptide derived from Emdogain ${ }^{\circledR}$ on human periodontal ligament 
fibroblasts. J Osaka Dent Univ 2009; 43: 111-7.

8. Taguchi Y, Yasui N, Tominaga K, et al. Usefulness of enamel matrix derivative-derived synthetic oligopeptides for human gingival epithelial cells. Jpn J Conserv Dent 2010; 53: 449-56. (Japanese)

9. Sabttini E, Bisgaard K, Ascani S, et al. The EnVisionTM + system: a new immunohistochemical method for diagnostics and research. Critical comparison with the APAAP, ChemMateTM, CSA, LABC, and SABC techniques. J Clin Pathol 1998; 51: 506-11.

10. Nakaya H, Kamoi K. Immunohistological study of wound healing in periodontal tissue of rats - Distribution of fibronectin and laminin after flap operation-. J Jpn Soc Periodontol 1989; 31: 462-90. (Japanese)

11. Sasano Y, Maruya Y, Sato H, et al. Distinctive expression of extracellular matrix molecules at mRNA and protein levels during formation of cellular and acellular cementum in the rat. Histochem J 2001; 33: 91-9.

12. D'errico JA, Ouyang H, Berry JE, et al. Immortalized Cementoblasts and periodontal ligament cells in culture. Bone 1999; 25: 39-47.

13. Sasano Y, Zhu J, Kamakura S, Kusunoki S, Mizoguchi I, Kagayama M. Expression of major bone extracellular matrix proteins during embryonic osteogenesis in rat mandibles. Anat Embryol 2000; 202: 31-7.

14. Jäger A, Kunert D, Friesen T, Zhang D, Lossdörfer S, Götz W. Cellular and extracellular factors in early root resorption repair in the rat. Eur J Orthod 2008; 30: 336-45.

15. D'errico JA, Macneil RL, Takata T, Berry J, Strayhorn C, Somerman MJ. Expression of bone associated markers by tooth root lining cells, in situ and in vitro. Bone 1997; 20: 117-26.

16. Bosshardt DD, Zalzal S, McKee MD, Nanci A.
Developmental appearance and distribution of bone sialoprotein and osteopontin in human and rat cementum. Anat Rec 1998; 250: 13-33.

17. MacNeil RL, Berry J, Strayhorn C, Somerman MJ. Expression of bone sialoprotein mRNA by cells lining the mouse tooth root during cementogenesis. Arch Oral Biol 1996; 41: 827-35.

18. Yamamoto T, Domon T, Takahashi S, Arambawatta AKS, Wakita M. Immunolocalization of proteoglycans and bonerelated noncollagenous glycoproteins in developing acellular cementum of rat molars. Cell Tissue Res 2004; 317: 299-312.

19. Kikuchi S. Experimental study of periodontal tissue regeneration after the application of enamel matrix derivative in rat periodontal defects. J Stomatol Soc Jpn 2001; 68: 82-92. (Japanese)

20. Paynter KJ, Pudy G. A study of the structure, chemical nature, and development of cementum in the rat. Anat Rec 1958; 131: 233-51.

21. Yamamoto T, Domon T, Takahashi S, Islam NMD, Suzuki R, Wakita M. The structure of the cemento-dentinal junction in rat molars. Ann Anat 2000; 182: 185-90.

22. Cheng H, Caterson B, Yamauchi M. Identification and immunolocalization of chondroitin sulfate proteoglycans in tooth cementum. Connect Tissue Res 1999; 40: 37-47.

23. Tominaga K, Tabata H, Kawanaka A, et al. Immunohistochemical stainability of rat maxillae and intestines immersed in six types of decalcifying solutions. J Osaka Dent Univ 2008; 42: 83-7.

24. Takahashi T, Ueda M. Effect of enamel matrix derivative on the formation of type I collagen during early wound healing of periodontal defects in rats. Shika Igaku (J Osaka Odontol Soc) 2007; 70: 42-8. (Japanese) 